\title{
Diffraction and rapidity gap measurements with ATLAS
}

\author{
Vlastimil Kus* \\ On behalf of the ATLAS Collaboration \\ Institute of Physics, Academy of Sciences of the CR \\ E-mail: vlastimil.kus@cern.ch
}

\begin{abstract}
Two diffraction related measurements of proton-proton collisions in the ATLAS experiment of the Large Hadron Collider at $\sqrt{ } s=7 \mathrm{TeV}$ centre-of-mass energy are presented: a measurement of relative diffractive contributions to the inelastic cross section in a diffractive enhanced event sample and a differential cross section of pseudo-rapidity gaps production using an early 2010 data sample. The rapidity gaps are defined as areas without any particles above different transverse momentum thresholds and are measured using the ATLAS tracking detector and calorimetry. Results are compared to several distinctive Monte Carlo models of diffraction and a constraint of the Pomeron intercept value in a triple Pomeron based approach is determined. Events with small pseudo-rapidity gaps region allow us to test different hadronisation models and statistical fluctuations of particle production during the hadronisation process.
\end{abstract}

International Conference on the Structure and the Interactions of the Photon including the 20th International Workshop on Photon-Photon Collisions and the International Workshop on High Energy Photon Linear Colliders

20 - 24 May 2013

Paris, France

\footnotetext{
*Speaker.
} 


\section{Introduction}

The total cross section in hadronic scattering experiments can be described as a sum of two components: elastic and inelastic, where the inelastic one can be furthermore divided into diffractive and non-diffractive (ND) contributions. This proceeding reports on two measurements performed by the ATLAS Collaboration. First, it is a measurement of the fraction of diffractive contribution to the inelastic cross section [1] $f_{D}$ which is compared to several different models. The second paper [2] presents a measurement of the rapidity gap cross section. Both measurements use small data sample of the integrated luminosity $<20 \mu \mathrm{b}^{-1}$.

Diffractive $p p$ interactions can be described in the realm of Regge theory [3], where perturbative calculations cannot be used. They are caused by $t$-channel color singlet exchange that can be represented by electroweak or strongly interacting states. It is this absence of color exchange that leads to a creation of so-called rapidity gaps, i.e. large areas in pseudo-rapidity devoid of any hadronic activity. This typical signature can be exploited by experimentalists to identify diffractive events.

As mentioned above, inelastic interactions consist of non-diffractive and diffractive contributions. The diffractive one can be further thought of as a composition of single diffractive dissociation (SD) $p p \rightarrow p X$ with one proton remaining intact and the other one producing a system $X$, double diffractive dissociation (DD) $p p \rightarrow X Y$ with both protons dissociating into typically low-mass final states $X$ and $Y$, and central diffractive contribution (CD) $p p \rightarrow p X p$ with both protons remaining intact and final state particles being produced in the central region. The rapidity gap is found in the forward region between the scattered proton and the system $X$ in SD case and in the central region between the systems $X$ and $Y$ in DD case. Two rapidity gaps in the forward region are found between the system $X$ and both protons for CD events. Besides these real diffractive events caused by an exchange of a colorless object (such as a Pomeron) between the interacting protons the rapidity gap can be also produced as a consequence of statistical fluctuations in the hadronisation process. These fake rapidity gaps are however exponentially suppressed [4] as a function of the gap size.

Basic kinematic variables commonly introduced to describe the diffractive dissociation are invariant masses of systems $X$ and $Y, M_{X}$ and $M_{Y}$ respectively, square four-momentum transfer $t$ and $\xi_{X}$ ( $\xi_{Y}$ respectively), that can be expressed in terms of invariant masses

$$
\xi_{X(Y)}=\frac{M_{X(Y)}^{2}}{s},
$$

where $s$ is the squared of the center-of-mass energy. In case of SD, variable $\xi_{X}$ corresponds to a fractional momentum loss of the final state proton. Moreover, the size of a rapidity gap is closely correlated with variable $\xi_{X}$

$$
\Delta \eta \approx-\ln \xi_{X}
$$

As perturbative calculations cannot be used for soft QCD interactions, the only available description is by phenomenological models such as Regge theory where the color singlet object is associated with the Pomeron [3]. Due to a freedom of choice among these models describing the diffractive final state, it is not clear which one is the best and that creates, along with the modelling 
of hadronisation, large uncertainties in model predictions which are visible in presented results. Therefore, these measurements are compared to several distinctive Monte Carlo models such as PYTHIA6 [5], PYTHIA8 [6], PHOJET [7] and HERWIG++ [8].

\section{Studies of diffractive enhanced minimum bias events}

In this measurement [1], the contribution of the diffractive component to the inelastic cross section was measured. Collision events were selected by the Minimum Bias Trigger Scintillator (MBTS) which is composed of two discs positioned symmetrically $3 \mathrm{~m}$ away from the interaction point covering pseudo-rapidity region $2.1<|\eta|<3$.8. A diffraction enhanced event sample was selected requiring MBTS to fire on one side of the interaction point only. Predictions show that, depending on the Monte Carlo model, 27-41\% of SD and DD events pass the single-sided selection whereas less than $1 \%$ of ND processes pass this single-sided criterion. The fractional diffractive contribution to $\sigma_{\text {inel }}$

$$
f_{D}=\frac{\sigma_{S D}+\sigma_{D D}}{\sigma_{N D}+\sigma_{S D}+\sigma_{D D}}
$$

is constrained by a ratio of single-sided processes to inclusive event sample $R_{S S}=N_{S S} / N_{\text {any }}$. The measured $R_{S S}$ in $\sqrt{ } s=7 \mathrm{TeV}$ data is

$$
R_{S S}=\left[10.02 \pm 0.03(\text { stat. })_{-0.4}^{+0.1}(\text { syst. })\right] \%,
$$

which is depicted in Fig. 1 and compared to different Monte Carlo models as a function of $f_{D}$. The predictions with variable $f_{D}$ were constructed keeping the contributions of SD and DD (and CD in case of PHOJET) fixed. The central value of $f_{D}$ for each model is then extracted as the intersection point with the measured $R_{S S}$, being $f_{D}=26.9_{-1.0}^{+2.5} \%$ for the default Donnachie-Landshoff (DL) model $(\varepsilon=0.085)$.

\section{Rapidity gap measurement}

The invariant mass of the dissociated system cannot be precisely measured unless the scattered proton is detected. This is due to the limited ATLAS detector [9] acceptance $(|\eta|<4.9)$. It can be, however, circumvented by measuring the gap size which is closely correlated with the invariant mass or $\xi$, as shown in Eq. 1.2 and 1.1. As explained above, for SD interactions gaps in the forward region are expected. We therefore introduce the $\Delta \eta^{F}$ variable quantifying the size of empty forward regions. They are defined as the largest size in $\eta$ ranging between one of the edges of the calorimeter $(\eta=-4.9$ or $\eta=+4.9)$ and particle (detector object) in the ATLAS acceptance. For a detector-level rapidity gap reconstruction, both the Inner Detector (providing us with trajectories of charged particles in $|\eta|<2.5$ region) and calorimeters are used. Highly segmented electromagnetic $(|\eta|<3.2)$, hadronic end-cap $(1.5<|\eta|<3.2)$ and forward $(3.1<|\eta|<4.9)$ calorimeters are used. As the rapidity gap measurement relies on the ability to identify large regions in the detector without any particle activity, well tuned noise-suppression cuts are crucial first to minimize mis-identifications due to noisy clusters of cells in calorimeters or inefficient track reconstruction, and second to avoid introducing fakes due to ignoring too many particles (i.e. an appropriate hadron level definition 


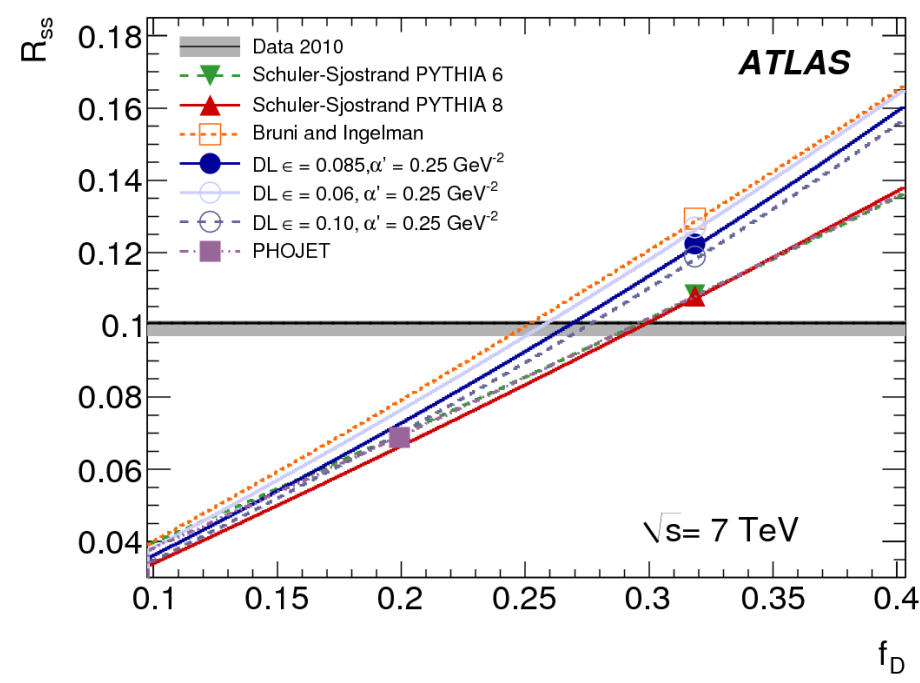

Figure 1: Ratio of single-sided (diffractive enhanced) to inclusive events, $R_{S S}$ as a function of fractional diffractive contribution to the inelastic cross section, $f_{D}$ [1]. The data value is shown with its systematic uncertainties (grey band) and is compared to several MC models.
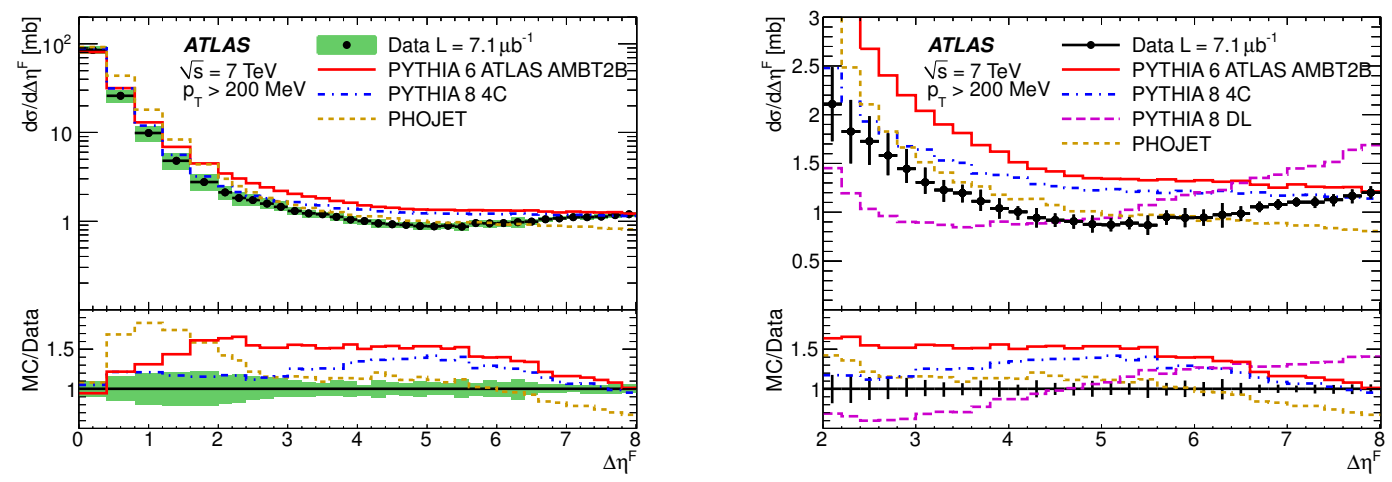

Figure 2: Rapidity gap distributions normalized to differential cross section. Comparison of different Monte Carlo models to data (left) in full $\Delta \eta^{F}$ region for particles with $p_{T}>200 \mathrm{MeV}$ and zoom into large gap region (right) [2].

needs to be derived corresponding to the detector level selection). Thanks to a careful noise cuts tuning one can minimize detector effects influencing the $\Delta \eta^{F}$ distribution. And it is this noise suppression requirement that leads to an exclusion of the hadronic tile calorimeter $(|\eta|<1.7)$ from the gap definition as its cell noise distributions contain non-Gaussian tails. For the other calorimeters a cut on cell significance $S=E / \sigma_{\text {noise }}$, where $E$ is the energy of a calorimeter cell and $\sigma_{\text {noise }}$ a standard deviation of Gaussian cell noise distribution, is used to separate a noise and a physical signal. There is an additional $p_{T}>200 \mathrm{MeV}$ cut on particles, clusters and tracks which was estimated as the smallest experimentally accessible transverse momentum. The measurement was also performed for different transverse momentum cut values, $200<p_{T}<800 \mathrm{MeV}$ (see [2]). 

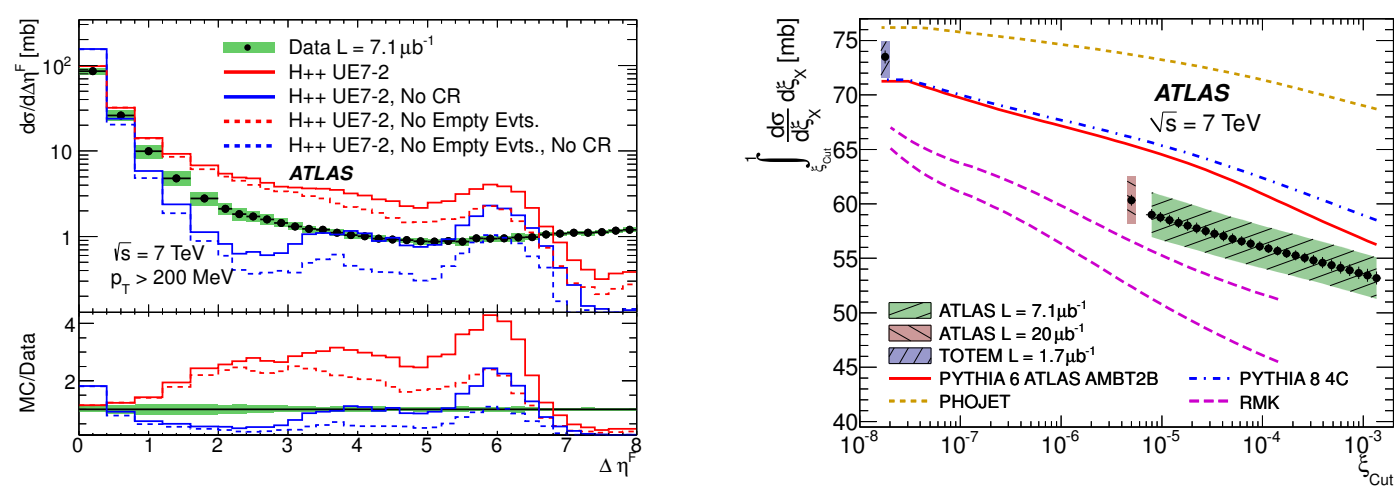

Figure 3: Rapidity gap distributions normalized to differential cross section (left) for HERWIG++ UE7-2 minimum bias model compared to data [2]. (Right) Inelastic cross section as a function of minimum $\xi$ cut [2].

Events used for this analysis come from an early 2010 data taking period. They were collected by the Minimum Bias Trigger Scintillator which is $~ 99 \%$ efficient for events with charged particles produced in its acceptance. The trigger requirement puts an upper limit on the measured size of rapidity gap $0<\Delta \eta_{F}<8$.

In Figure 2, the differential inelastic cross section as a function of forward pseudo-rapidity gaps, $\Delta \eta^{F}$, is presented and compared to different Monte Carlo predictions. Systematic uncertainties, dominated by Monte Carlo modelling, are typically below $\sim 8 \%$ for large gaps $\left(\Delta \eta^{F}\right)$ growing up to $\sim 20 \%$ at $\Delta \eta^{F} \sim 1.5$. Two distinctive features of diffractive interactions can be observed. First, there is an exponential decrease in the beginning of the spectrum (ND dominated region according to all MCs) caused by a steeply falling probability of hadronisation fluctuations producing large gaps. This region is best described by PYTHIA8 model, while PHOJET overshoots data almost by a factor of two due to wrong ND normalization. Secondly, it is a saturation called diffractive plateau that is reached around $\Delta \eta^{F} \sim 3$ and where the SD+DD events are a dominant contribution according to models presented in this figure. The right plot of Fig. 2 zooms into large gap region and reveals a growth of the differential cross section at very large gaps in data as well as in PYTHIA8 with the Donnachie-Landshoff model which uses the default Pomeron trajectory, $\alpha_{\mathbb{P}}(t)=1.085+0.25 t$. Though the data at large $\Delta \eta^{F}$ are not described well by this choice, it can be used in its cleanest diffractive region $\left(\Delta \eta^{F}>6\right)$ to get a best estimate, by means of a fit, of the Pomeron intercept. The obtained value is

$$
\alpha_{\mathbb{P}}(0)=1.058 \pm 0.003 \text { (stat.) }{ }_{-0.039}^{+0.034} \text { (syst.). }
$$

Since rapidity gap distributions are sensitive to fluctuations in hadronisation processes, they can be used to evaluate different hadronisation models such as the Lund String model used in PYTHIA and PHOJET or an alternative cluster hadronisation model implemented in HERWIG++. Figure 3 presents a comparison of HERWIG++ Minimum Bias model tune UE7-2 to data in the left plot. Though it does not contain diffractive processes and thus exponential ND decrease is expected, HERWIG++ clearly produces significant large gaps and a bump around $\Delta \eta^{F}=6$. This behaviour is 
present even with Color Reconnection switched off or excluding events with no scatterings of the soft or semi-hard types, for which the contribution of large rapidity gaps is expected to be smaller.

Due to the correlation between $\Delta \eta^{F}$ and $\xi$ variables as demonstrated by Eq. 1.2 it is possible to calculate the inelastic cross section integrated from certain minimal $\xi$ value (denoted as $\xi_{C u t}$ ) up to 1 , which is equivalent to integrating cross section as a function of rapidity gap size in $0<\Delta \eta^{F}<$ $\Delta \eta_{\max }^{F}$ range. Results from the measurement [2] are compared with previous ATLAS results [10] and the TOTEM measurement integrated over all accessible $\xi_{X}$ region [11]. Data is compared to standard MC models as well as to two versions of the KMR model [12]. While PYTHIA and PHOJET fail to describe the data, the KMR model - though with incorrect normalization - describes the shape of the data better than the other ones. This indicates that the size of the low-mass diffraction is underestimated in most of the conventional models.

\section{References}

[1] ATLAS Collaboration, Measurement of the Inelastic Proton-Proton Cross Section at $\sqrt{ } s=7 \mathrm{TeV}$ with the ATLAS Detector, Nature Commun. 2 (2011) 463

[2] ATLAS Collaboration, Rapidity gap cross sections measured with the ATLAS detector in pp collisions at $\sqrt{ } s=7 \mathrm{TeV}$, Eur.Phys.J. C72 (2012) 1926

[3] G. Chew, S. Frautschi, Phys. Rev. Lett., 7 (1961) 394

[4] J. Bjorken, S. Brodsky, H. J. Lu, Phys. Lett., B286 (1992) 153

[5] T. Sjostrand et al., PYTHIA 6.4 physics and manual, arXiv:0603175 [hep-ph]]

[6] T. Sjostrand, S. Marenna and P. Skands, Comput. Phys. Commun. 178 (2008) 852

[7] R. Engel, Z. Phys.C66 (1995) 203

[8] M. Bahr et al., Herwig++ Physics and Manual, Eur. Phys. J.C58 (2008) 639-707

[9] ATLAS Collaboration, The ATLAS Experiment at the CERN Large Hadron Collider, JINST 3 (2008) S08003

[10] ATLAS Collaboration, Nature Comm. 2 (2011) 463

[11] TOTEM Collaboration, Europhys. Lett. 96 (2011) 21002

[12] M. G. Ryskin, A. D. Martin, V. A. Khoze, Eur. Phys. J., C71 (2011) 1617 